\title{
Physico-Chemical and Chromatic Characterization of Malvidin 3-Glucoside-vinylcatechol and Malvidin 3-Glucoside-vinylguaiacol Wine Pigments
}

\author{
Natalia Quijada-Morín, ${ }^{\dagger}$ Olivier Dangles, ${ }^{*}$ Julián C. Rivas-Gonzalo, ${ }^{\dagger}$ and \\ M. Teresa Escribano-Bailón $*, \dagger$ \\ †Grupo de Investigación en Polifenoles, Universidad de Salamanca, E-37007 Salamanca, Spain, and \\ *Université d'Avignon et des Pays de Vaucluse, INRA, UMR408, F-84000 Avignon, France
}

\begin{abstract}
The physicochemical and chromatic features of malvidin 3-glucoside-vinylcatechol (PA1) and malvidin 3-glucoside-vinylguaiacol (PA2) adducts were investigated. Important differences between both pigments were observed. In the investigated $\mathrm{pH}$ range (2.0-4.5), our results suggest that PA1 could be considered as a noncovalent dimer of two pyranoflavylium ions $(\mathrm{AH})_{2}$ which undergo a hydration reaction in two successive steps, with no proton transfer. In contrast, only proton transfer equilibrium between the pyranoflavylium ion and the quinonoid bases was observed for PA2. The hydration and acidity thermodynamic constants of both pyranoanathocyanins were determined by UV-vis spectroscopy. Pigment PA1 was shown to be less sensitive to bisulfite addition than to water addition, and PA2 seems to be largely insensitive to both water and bisulfite additions. The binding constants for the interaction between the pigments and (+)-catechin in aqueous solution and the changes in the CIELAB parameters that it provoked were also determined. The huge increase in the absorption of the pyranoflavylium ion of PA2 when it binds catechin has no equivalent for anthocyanins and nicely outlines the potential of pyranoanthocyanins in the expression of natural colors.
\end{abstract}

KEYWORDS: Pyranoanthocyanin; red wine pigments; copigmentation; malvidin 3-O-glucoside; hydroxycinnamic acid; caffeic acid; ferulic acid; CIELAB

\section{INTRODUCTION}

Anthocyanins are responsible for the purple-red color of young red wines. During aging the color shifts to a more reddish brown hue, mainly due to the progressive replacement of anthocyanins by more stable pigments $(1-3)$. Different types of anthocyanin derivatives could be involved in these color changes, in particular pyranoanthocyanins $(4,5)$. These pigments are formed by the cycloaddition of diverse wine nucleophiles at $\mathrm{C} 4$ and $\mathrm{O} 5$ of the flavylium nucleus (followed by aromatization through autoxidation), thus originating an additional pyrane ring in the pigment structure. Pyruvic acid $(6,7)$, acetaldehyde $(8,9)$, vinylphenol $(10)$, vinylcatechol (11), vinylguaiacol (2), flavanol monomers and dimers $(12,13)$, acetone $(9,14)$, and acetoacetic acid $(15)$ have been demonstrated to react with anthocyanin originating pyranoanthocyanins, which have been detected in red wines. The cycloaddition reaction causes a hypsochromic shift in the visible absorption maximum of the chromophore, thus changing wine color toward more orange hues $(6-14)$. Also, colors expressed by pyranoanthocyanin typically display a higher stability compared to that of the original anthocyanins $(6,7)$.

The formation of vinylphenol, vinylcatechol, and vinylguaiacol derivatives during wine aging can occur through two different

*To whom correspondence should be addressed. Phone: +34923 294 537. Fax: +34 923294 515. E-mail: escriban@usal.es. mechanisms. On the one hand, Fulcrand et al. (10) stated that the malvidin 3-glucoside/4-vinylphenol adduct can be formed from vinylphenol released in wine by enzymatic decarboxylation of $p$-coumaric acid. It has been suggested that the formation of the vinylcatechol and vinylguaiacol derivatives follows a similar mechanism (2). On the other hand, Schwarz et al. (16) have demonstrated that these pigments can also be produced in the reaction between the anthocyanin and the intact hydroxycinnamic acid. During the first months of aging, the first mechanism would take place while the second mechanism, which is much slower, would take place over longer periods of aging (17).

The structural transformations of anthocyanins in aqueous solution are well known $(18-20)$. Depending on the $\mathrm{pH}$, they exist in the form of different species in equilibrium through hydration, proton transfer, and tautomerization reactions. In very acidic aqueous solutions, the red flavylium ion is predominant. As $\mathrm{pH}$ increases, the nucleophilic attack of water at position 2 of the pyrylium nucleus gives rise to the colorless hemiketal (thermodynamic product), which is in equilibrium with minor concentrations of chalcones. Concurrently, deprotonation of the flavylium cation also occurs, leading to blue-purple quinonoid bases (kinetic products), which are typically in low concentration at equilibrium. The distribution among these colored and colorless forms also depends on the temperature, composition of the medium, and the molecular interactions that may develop 
between anthocyanins and other phenols ( $\pi$-stacking). These interactions, named copigmentation, are much stronger with the polarizable planar colored forms than with the hemiketal and thus protect the flavylium chromophore against the nucleophilic attack of water $(21,22)$. While the structural transformations of anthocyanins are well known, few results have been reported on the physicochemical properties of anthocyaninderived pigments $(23-25)$.

The aim of this work was to better understand the contribution of the pyranoanthocyanins to the color of aged red wines. For this purpose, the color characteristics, stability in relation to $\mathrm{pH}$ changes, discoloration by $\mathrm{SO}_{2}$, and storage in aqueous solution of the 4-vinylguaiacol and 4-vinylcatechol adducts of malvidin 3 -glucoside were studied. The structural transformations in acidic media and copigmentation with the common wine copigment $(+)$-catechin were also investigated.

\section{MATERIALS AND METHODS}

Materials. Malvidin 3-O-glucoside (M3G) was isolated in the laboratory from skins of Vitis vinifera red grapes of the Tempranillo variety, by extraction with acidic methanol and further purification by medium-pressure liquid chromatography using a reversed-phase column, as described by Heredia et al. (26). The identity and purity were checked by HPLCDAD-MS by comparing it with a commercial standard. The M3G standard was purchased from Polyphenols Laboratoires A.S. Sandnes, Norway. Caffeic acid, ferulic acid, and (+)-catechin were purchased from Sigma-Aldrich Chemie (Germany). All solvents were of HPLC quality.

Synthesis of the 4-Vinylcatechol-M3G and 4-Vinylguaiacol-M3G Adducts. The hemisynthesis of phenyl-substituted pyranoanthocyanins from malvidin 3-glucoside (M3G) and hydroxycinnamic acids was carried out using a simple one-step procedure already described for the formation of phenyl-substituted pyranoanthocyanins (16). Aqueous solutions of $\mathrm{M} 3 \mathrm{G}$ were incubated at $\mathrm{pH} 0.5$ at $35^{\circ} \mathrm{C}$ with caffeic acid or with ferulic acid (hydroxycinnamic acid/M3G molar ratio of 20:1). The formation of new pigments was monitored by HPLC-DAD-MS. After three weeks, the 4-vinylcatechol-M3G adduct PA1 and 4-vinylguaiacol-M3G adduct PA2 were isolated from their respective solutions.

Purification of PA1 and PA2. The adducts were isolated and purified by semipreparative HPLC using Waters 600 equipment and an Ascentis C18 $5 \mu \mathrm{m}(250 \times 10 \mathrm{~mm})$ column (Sigma Aldrich-Supelco, St. Louis, MO, USA). The solvents were TFA $0.1 \%$ (A) and acetonitrile (B). The elution profile was as follows: isocratic $10 \%$ B for $3 \mathrm{~min}$, from 10 to $18 \%$ B for $3 \mathrm{~min}$, from 18 to $30 \%$ B for $15 \mathrm{~min}$, from 30 to $35 \%$ B for $5 \mathrm{~min}$, and from 30 to $50 \%$ B for $5 \mathrm{~min}$, at a flow rate of $3 \mathrm{~mL} / \mathrm{min}$. Detection was carried out at $505 \mathrm{~nm}$, and the peaks were collected in a fraction collector. Solvents were eliminated under vacuum, and the compound was transferred to water and freeze-dried in a Telstar Cryodos-80. The purity and identity of the pigments isolated were checked by HPLC-DAD and MS. When the purified PA1 and PA2 were separately injected in the HPLC-DAD system, no other peaks were detected in the $250-600 \mathrm{~nm}$ range. PA1 and PA2 had the same retention time and $\mathrm{UV}$-vis spectrum that those reported in wine for the vinylcatechol derivative of $\mathrm{M} 3 \mathrm{G}$ and vinylguaiacol derivative of M3G (17), respectively. The molecular ion of PA1 had the same $m / z$ ratio $\left(m / z\right.$ 625) and yielded the same fragment ion in the $\operatorname{MS}^{2}(m / z 463)$ as the 4-vinylcatechol adduct of M3G reported in wines $(2,17,27-29)$. The molecular ion of PA2 yielded a signal in the mass spectrum at $m / z 639$, and in the $\mathrm{MS}^{2}$ analysis, it was fragmented into a single ion with an $\mathrm{m} / \mathrm{z}$ ratio at 477 , which was consistent with the data reported in the literature $(2,17,27,29)$.

HPLC-DAD-MS Analysis. HPLC-DAD analysis was performed in a Hewlett-Packard 1100 series liquid chromatograph. An Aqua C18 reverse phase, $5 \mu \mathrm{m}, 150 \mathrm{~mm} \times 4.6 \mathrm{~mm}$ column (Phenomenex,Torrance, CA, USA) thermostatted at $35^{\circ} \mathrm{C}$, was used. The mass analyses were performed using a FinniganTM LCQ ion trap instrument (Thermoquest, San Jose, CA, USA) equipped with an electrospray ionization (ESI) interface. The LC system was connected to the probe of the mass spectrometer via the UV cell outlet. HPLC-DAD-MS conditions were as described elsewhere (27).
Thermodynamic Measurements. Solutions of pigments PA1 and PA2 $\left(10^{-4} \mathrm{M}\right)$ were prepared in a citrate buffer $(0.1 \mathrm{M})$ at different $\mathrm{pH}$ values ranging from 1.0 to 4.5 (ionic strength adjusted to $1 \mathrm{M}$ by $\mathrm{NaCl}$ ). The solutions were kept for $2 \mathrm{~h}$ at $20{ }^{\circ} \mathrm{C}$ to reach complete hydration equilibrium prior to the spectrophotometric measurement. Absorption spectra were recorded using a Hewlett- Packard UV-vis HP8453 spectrophotometer (Palo Alto, CA).

Bleaching by $\mathrm{SO}_{2}$. The solutions used in the thermodynamic measurements of each pigment were separately collected and adjusted to $\mathrm{pH}$ 3.5 with a more acidic pigment solution at the same concentration $\left(10^{-4}\right.$ M). Forty microliters of aqueous solutions of sodium bisulfite at concentrations of 0,5 , and $10 \mathrm{mg} / \mathrm{mL}$ was added to $2 \mathrm{~mL}$ of each pigment solution to achieve total $\mathrm{SO}_{2}$ concentrations of 0,100 , and $200 \mathrm{ppm}$. The solutions were left to equilibrate for $15 \mathrm{~min}$ before spectrophotometric measurement. Absorption spectra were recorded using a Hewlett- Packard UV-vis HP8453 spectrophotometer (Palo Alto, CA).

Colorimetric Measurements. The absorption spectra were recorded on a Hewlett-Packard UV-visible HP 8453 spectrophotometer (Palo Alto, CA), at $25^{\circ} \mathrm{C}$ using $10 \mathrm{~mm}$ path length glass cells. The whole visible spectrum $(380-770 \mathrm{~nm})$ was recorded $(\Delta \lambda=2 \mathrm{~nm})$, and Illuminant D65 and $10^{\circ}$ Observer were used in the calculations. The CIELAB parameters $\left(L^{*}, a^{*}, b^{*}, C^{*}{ }_{\mathrm{ab}}\right.$, and $h_{\mathrm{ab}}$ ) were determined by using the original software CromaLab (30), following the recommendations of the Commission International de L'Éclairage (31).

Copigmentation Experiments. Copigmented aqueous solutions of each pigment were prepared at $\mathrm{pH} 3.5$ (ionic strength adjusted to $1 \mathrm{M}$ ). Each solution contained the same pigment concentration $\left(10^{-4} \mathrm{M}\right)$ and increased concentrations of $(+)$-catechin so as to obtain a copigment/ pigment molar ratio ranging from 0 to 100 .

Relative Stability of PA1 and PA2 in Aqueous Solutions at $32{ }^{\circ} \mathrm{C}$. Solutions of both pigments $\left(10^{-4} \mathrm{M}\right)$ were prepared in a citrate buffer at $\mathrm{pH} 3.5$ and maintained at $32{ }^{\circ} \mathrm{C}$ in closed vials in the dark. Samples were taken up weekly, and changes were monitored by HPLC-DAD-MS. Data were compared with those of M3G obtained from the literature (32).

Molar Absorption Coefficients. Solutions of PA1 and PA2 in concentrations ranging from $2.4 \times 10^{-6}$ to $1.6 \times 10^{-4} \mathrm{M}$ for PA1 and $3.0 \times 10^{-6}$ to $1.0 \times 10^{-4} \mathrm{M}$ for PA2 were prepared in a citrate buffer adjusted to $\mathrm{pH} 0.8$ and $1 \mathrm{M}$ ionic strength. Solutions were left to equilibrate for $2 \mathrm{~h}$ before the determination of absorbance at $\lambda_{\max }: 507 \mathrm{~nm}$ for PA1 and $508 \mathrm{~nm}$ for PA2 and $22{ }^{\circ} \mathrm{C}$.

Data Analysis. Semiempirical quantum mechanics calculations were performed with the Hyperchem software (Autodesk, Sausalito, USA) using the PM3 program. The Scientist software (MicroMath, Salt Lake City, USA) was used for all curve-fitting procedures. The equations of regression used for calculating the molar absorption coefficients were obtained by plotting the absorbance values versus pigment concentration. The goodness of fit of the regression model was evaluated by the F-test using SPSS 13.0 statistical software.

\section{RESULTS AND DISCUSSION}

Pigment Preparation and Molar Absorption Coefficients. The linearity of the regression models used for calculating the molar absorption coefficients of PA1 and PA2 at the wavelength of maximal visible absorption $\left(\varepsilon_{\max }\right)$ was evaluated. For this, the regression variance and residual variance were obtained by means of ANOVA analysis. The $p$-values in the F-test were lower than 0.05 , indicating statistically valid fit. Therefore, absorbance versus concentration plots proved to be linear in the selected concentration ranges (see Materials and Methods section). This indicates the absence of deviations from Beer's law and consequently suggests that self-association either does not take place or is not significantly affected by dilution in the selected concentration ranges. The $\varepsilon_{\max }$ values at $\mathrm{pH} 0.8$ were $4496( \pm 48) \mathrm{M}^{-1} \mathrm{~cm}^{-1}$ $\left(\lambda_{\max }=507 \mathrm{~nm}\right)$ and $3058( \pm 46) \mathrm{M}^{-1} \mathrm{~cm}^{-1}\left(\lambda_{\max }=508 \mathrm{~nm}\right)$ for PA1 and PA2, respectively. These values were much lower than that previously determined for M3G (20200 $\mathrm{M}^{-1} \mathrm{~cm}^{-1}$ ) (26). Vinylguaiacol derivatives are found in low concentration in wine (17). This could be due to the low concentration of the ferulic acid precursor in wine and the higher reactivity of 


\section{PA1}

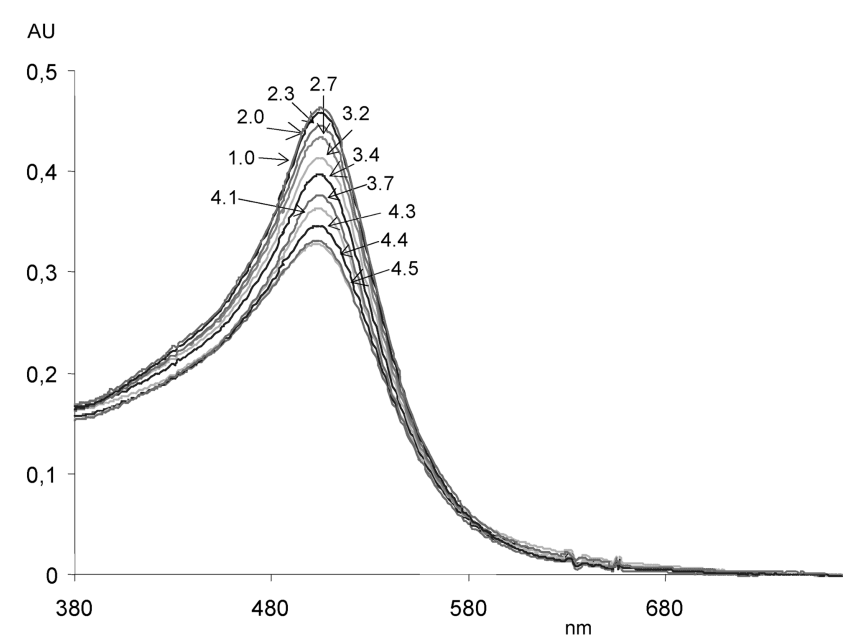

PA2

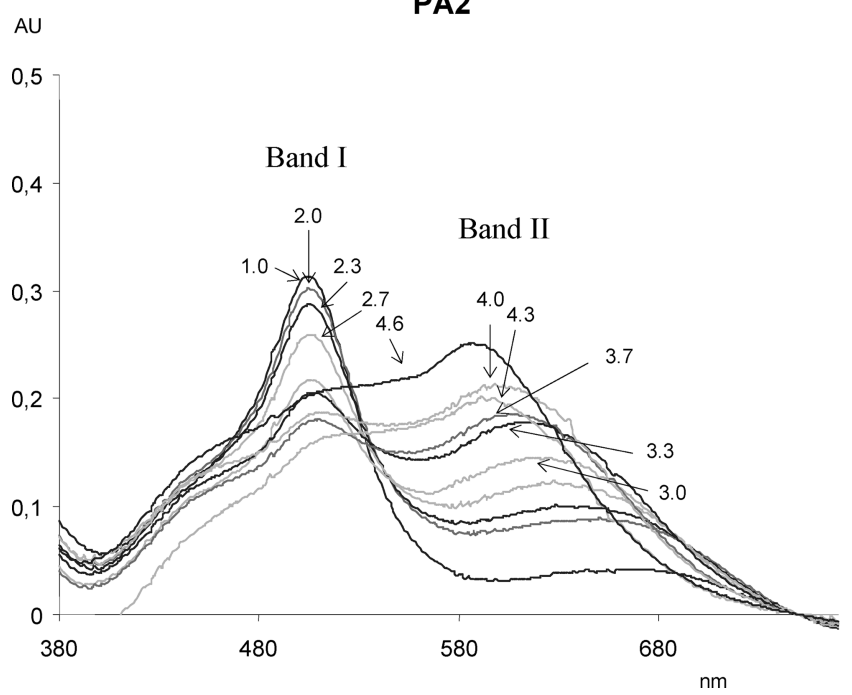

Figure 1. UV-visible spectra of PA1 and PA2 at different $\mathrm{pH}$ values (concentration of pigments, $10^{-4} \mathrm{M}$ ).

anthocyanins toward other hydroxycinnamic acids (observed in our laboratory and previously pointed out in ref 16 ). The low $\varepsilon_{\max }$ value of PA2 could also hinder its detection in wine.

The molar absorption coefficient of PA2 is reported for the first time in this article. The $\varepsilon_{\max }$ value of the vinylcatechol adduct (PA1) was already determined by Håkansson et al. (11), but in that article, the concentration range selected for its determination was not specified. Also, the experimental conditions (model wine solutions at $\mathrm{pH} 1.5$ and 3.6) were different from ours. Hence, it is difficult to compare both results.

Effect of $\mathrm{pH}$ and $\mathrm{SO}_{2}$ on Color Stability. Figure 1 shows the $\mathrm{pH}$ dependence of the visible spectra of PA1 and PA2 in aqueous solution. Important differences between both pigments can be observed. As the $\mathrm{pH}$ increases from 1 to 4.5, the visible spectrum of PA1 undergoes a clear hypochromic effect without any change in the $\lambda_{\max }$ value. The noteworthy decrease in the absorbance can be attributed to the formation of the colorless hemiketal form. By contrast, proton transfer does not take place since no bathochromic shift is observed (33). As expected, the hypochromic effect is less intense than with M3G (26). Indeed, substitution at $\mathrm{C} 4$ of the pyrylium $\mathrm{C}$-ring protects the chromophore from the attack of nucleophiles such as water or bisulfite $(34,35)$.

Unexpectedly, two absorption bands can be observed in the spectrum of PA2 at $\mathrm{pH} 1$. An intense one (band I) at $\lambda_{\max }=508 \mathrm{~nm}$,
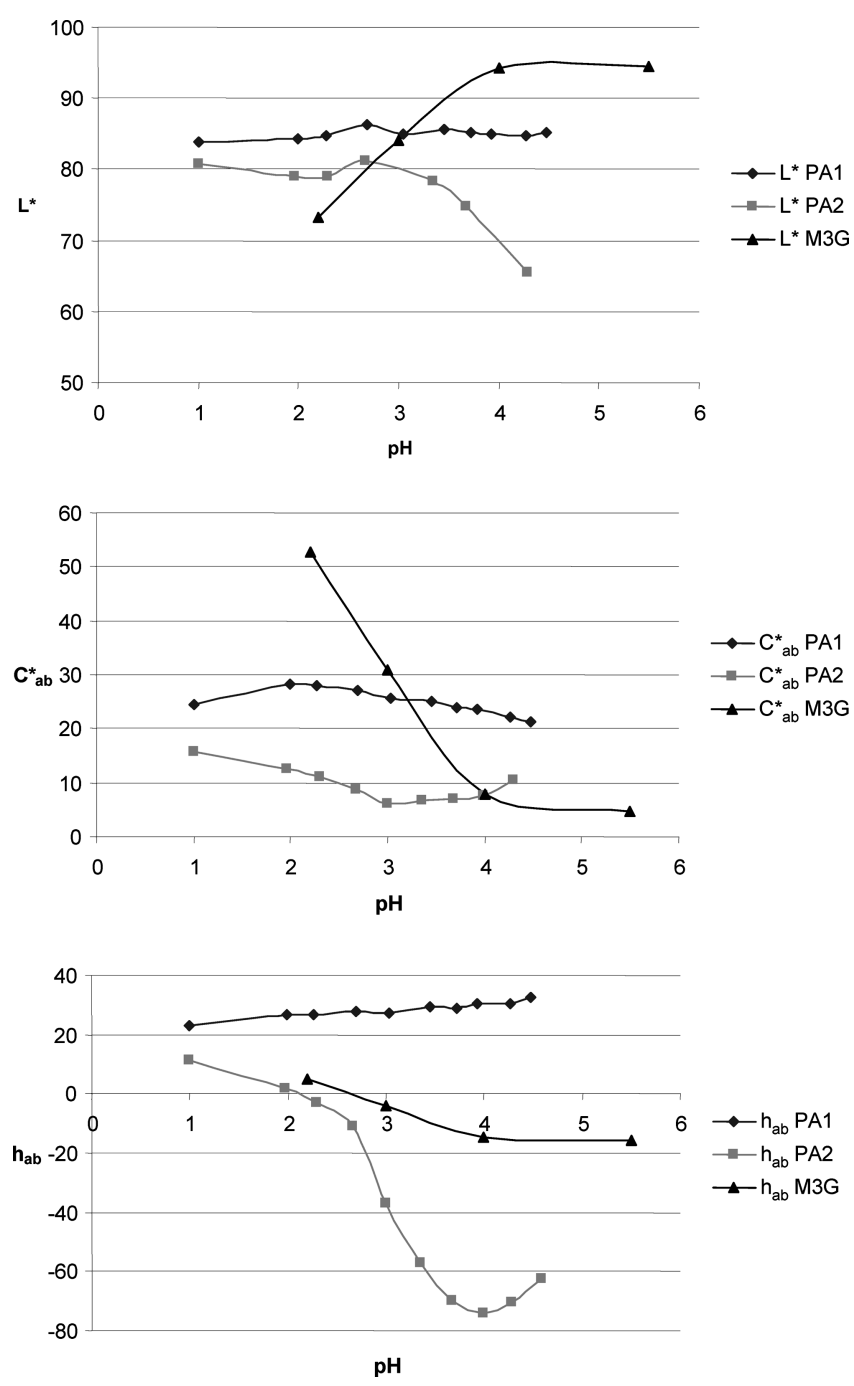

Figure 2. Changes in the lightness $\left(L^{*}\right)$, chroma $\left(C^{*}{ }_{a b}\right)$, and hue angle $\left(h_{\mathrm{ab}}\right)$ of solutions containing PA1, PA2, and M3G at different $\mathrm{pH}$ values.

which is characteristic of pyranoanthocyanins, and a much weaker one (band II) at $\lambda_{\max } \approx 670 \mathrm{~nm}$. As the $\mathrm{pH}$ increases, band I undergoes a decrease in absorbance with no significant change in $\lambda_{\max }$, while band II is strongly intensified with a concomitant hypsochromic shift. The formation of this new chromophore suggests the deprotonation of the pyranoflavylium ion in the $\mathrm{pH}$ range studied. Similar observations were reported with 8,8-methylmethine catechin-M3G pigments (24).

Figure 2 shows the $\mathrm{pH}$ dependence of $L^{*}, C^{*}$ ab, and $h_{\mathrm{ab}}$ for $\mathrm{M} 3 \mathrm{G}$ and the pyranoanthocyanins. As the $\mathrm{pH}$ increases, the large color loss in the $\mathrm{M} 3 \mathrm{G}$ solution results in an important increase of lightness and a decrease of chromaticity. In addition, a change to blue hues is observed, probably due to the formation of the quinonoid bases in low concentration (36). In contrast, the fading of the PA1 solution is much less noticeable and an increase of 10 CIELab units in the hue angle toward more orange hues suggests the appearance of yellow-orange forms, which could be chalcones in low concentration.

By contrast, the increase of $\mathrm{pH}$ in the $\mathrm{PA} 2$ solution provokes a decrease in lightness and therefore a darkening of the pigment solution. Moreover, a spectacular decrease of the hue angle occurs ( 70 CIELab units), indicating the possible presence of blue quinonoid forms. Chroma values decrease until $\mathrm{pH} 3$ and then tend to increase again.

The effect of $\mathrm{SO}_{2}$ on the color of pyranoanthocyanin solutions was studied at $\mathrm{pH} 3.5$ (usual wine $\mathrm{pH}$ ). Only a very small 

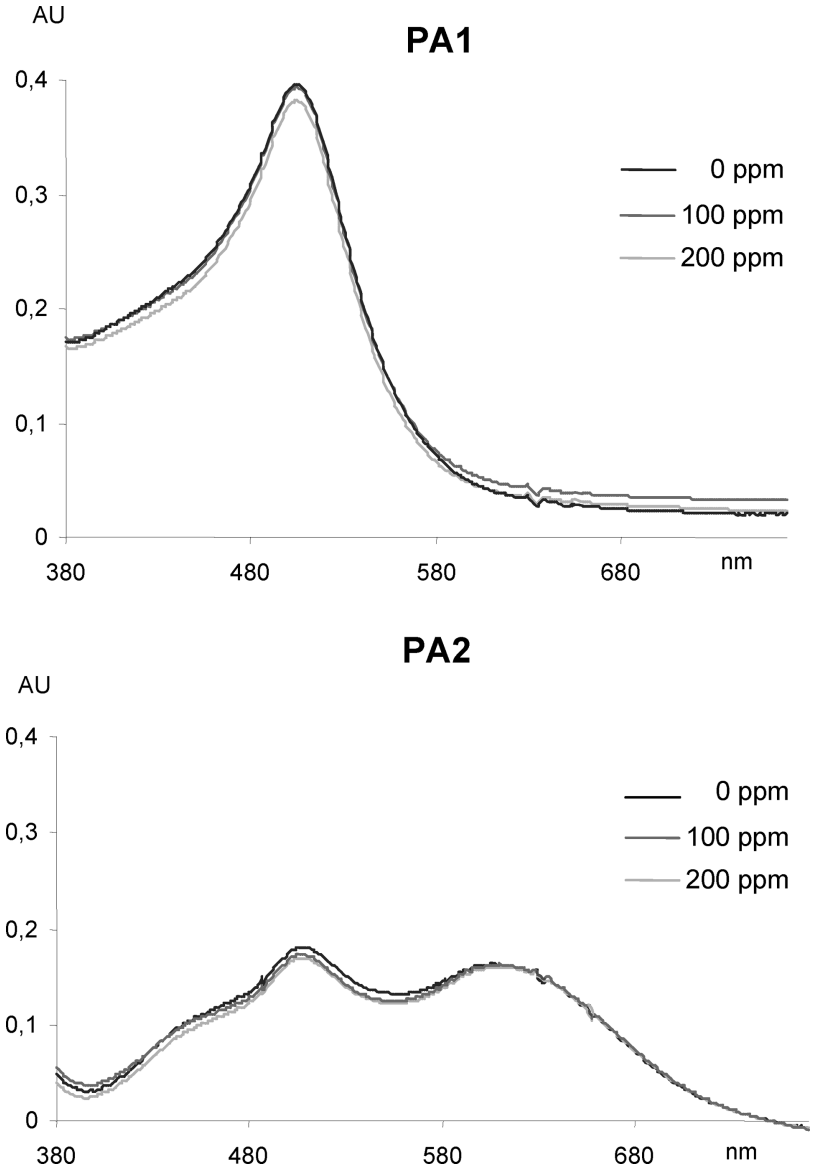

Figure 3. Effects of the addition of increasing concentrations of $\mathrm{SO}_{2}$ on the visible spectra of PA1 and PA2.

Table 1. $L^{*}, h_{\mathrm{ab}}$, and $C^{*}$ ab Values in the CIELAB Color Space of PA1, PA2, and M3G Solutions with the Addition of Increasing Concentrations of $\mathrm{SO}_{2}$

\begin{tabular}{|c|c|c|c|c|c|c|c|c|c|}
\hline \multirow[b]{2}{*}{$\mathrm{ppm} \mathrm{SO} \mathrm{S}_{2}$} & \multicolumn{3}{|c|}{ PA1 } & \multicolumn{3}{|c|}{ PA2 } & \multicolumn{3}{|c|}{ M3G } \\
\hline & $L^{*}$ & $C_{a b}^{\star}$ & $h_{\mathrm{ab}}$ & $L^{*}$ & $C^{*}{ }_{a b}$ & $h_{\mathrm{ab}}$ & $L^{*}$ & $C^{*}{ }_{a b}$ & $h_{\mathrm{ab}}$ \\
\hline 0 & 86 & 24 & 27 & 78 & 6 & -60 & 73 & 53 & $\overline{5}$ \\
\hline 100 & 86 & 23 & 26 & 79 & 6 & -66 & 93 & 14 & -11 \\
\hline 200 & 87 & 23 & 27 & 82 & 6 & -67 & 96 & 8 & -18 \\
\hline
\end{tabular}

hypochromic effect was observed (Figure 3). Moreover, the $\mathrm{SO}_{2^{-}}$ induced color loss of the PA2 solution reflects a very weak decrease in band I $(505 \mathrm{~nm})$, with band II $(\sim 590 \mathrm{~nm})$ remaining unaffected. The addition of $\mathrm{SO}_{2}$ also promotes a decrease of 7 CIELab units in the $h_{\mathrm{ab}}$ value, indicating a slightly bluer hue (Table 1). Also, a slight increase in the lightness of the solutions was detected.

These results suggest that PA1 and PA2 are largely insensitive to bisulfite addition. This is consistent with the regioselectivity of nucleophilic additions on flavylium ions, i.e., addition at $\mathrm{C} 2$ for water and addition at $\mathrm{C} 4$ for bisulfite (37). Indeed, C4 is substituted in pyranoflavylium ions, while $\mathrm{C} 2$ remains available for water addition, which may also take place on the additional heterocycle (Figure 4).

Thermodynamics of Hydration and Proton Transfer Reactions. In the PA1 solution, the decrease of the visible absorbance and the absence of bathochromic shift in the investigated $\mathrm{pH}$ range both suggest a pure hydration reaction with no proton transfer taking place. However, when the plot of the absorbance at $507 \mathrm{~nm}$ versus $\mathrm{pH}$ (Figure 5) was analyzed by assuming a simple water addition on the pyranoflavylium ion, no correct curve-fitting could be obtained (poor correlation coefficient; data not shown). In fact,

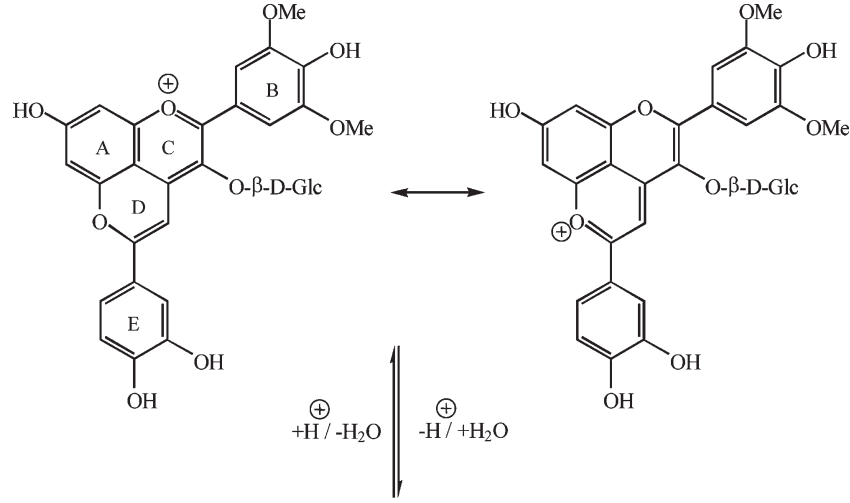

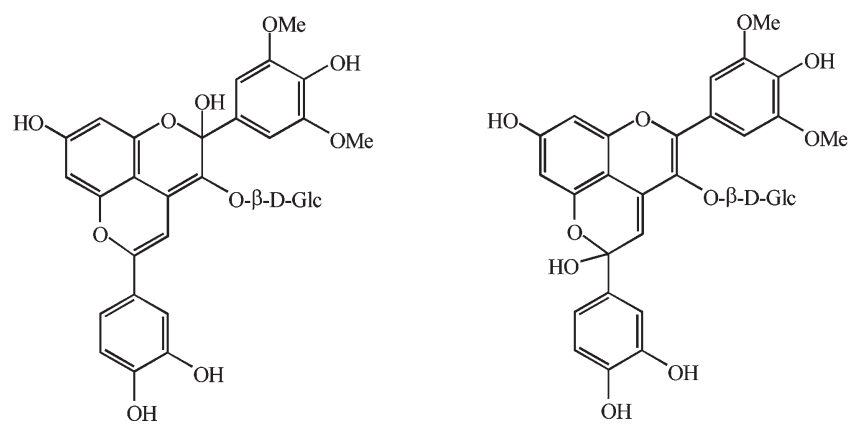

Figure 4. Water addition on PA1.

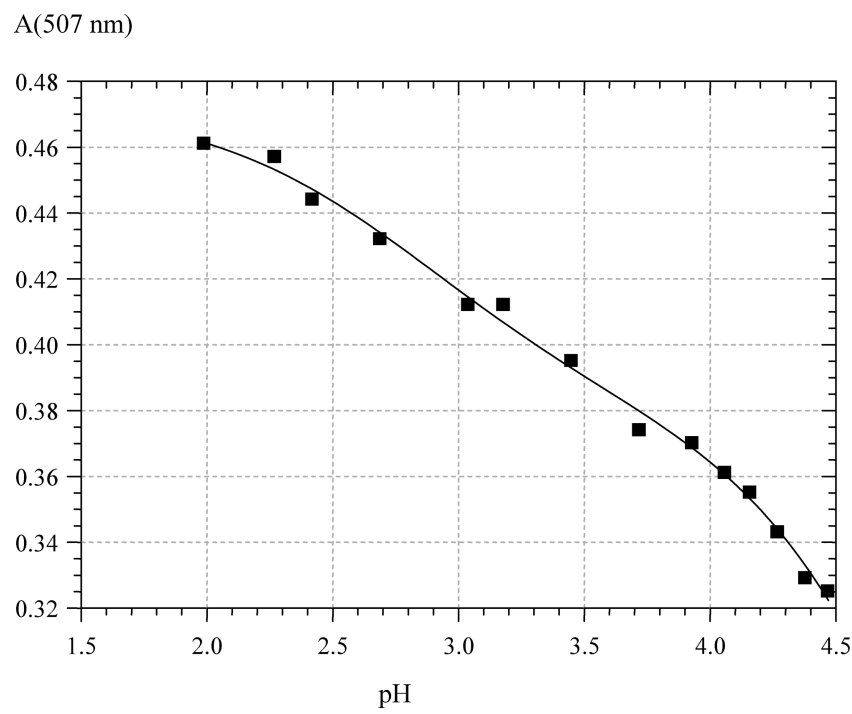

Figure 5. Changes in the absorbance at $507 \mathrm{~nm}$ of PA1 solutions at different $\mathrm{pH}$ values (concentration of pigments, $10^{-4} \mathrm{M}$ ).

the color loss seems to occur in two successive hydration steps. This unusual behavior was already observed with specific wine pigments in which $\mathrm{M} 3 \mathrm{G}$ is linked to a catechin moiety via an ethyl bridge derived from ethanal (itself formed by ethanol oxidation). It was interpreted by assuming that such pigments are especially prone to noncovalent dimerization ( $\pi$-stacking) and that the pigment molecules within the dimer undergo sequential hydration $(23,24)$. A similar analysis was successfully applied to PA1 regarded as a noncovalent assembly of two pigment molecules $(\mathrm{AH})_{2}$ (hydration thermodynamic constants $K_{\mathrm{h} 1}$ and $K_{\mathrm{h} 2}$ ):

$$
\begin{gathered}
(\mathrm{AH})_{2}+\mathrm{H}_{2} \mathrm{O} \leftrightarrow \mathrm{AHB}+\mathrm{H}^{+}, K_{\mathrm{h} 1} \\
\mathrm{AHB}+\mathrm{H}_{2} \mathrm{O} \leftrightarrow \mathrm{B}_{2}+\mathrm{H}^{+}, K_{\mathrm{h} 2}
\end{gathered}
$$

where $(\mathrm{AH})_{2}$, colored homodimer (two pyrylium nuclei); $\mathrm{AHB}$, heterodimer (1 pyrylium nucleus +1 hemiketal nucleus, 
$\left.r=\varepsilon_{\mathrm{AHB}} / \varepsilon_{\mathrm{AH} 2}<1\right)$; and $\mathrm{B}_{2}$, colorless homodimer $(2$ hemiketal nuclei). A combination of Beer's law, mass conservation for the pigment, and mass law readily gives eq 1 , which is used in the calculations $\left(A_{0}=\right.$ visible absorbance of a strongly acidified solution of pure $\left.(\mathrm{AH})_{2}\right)$ :

$$
A=A_{0} \frac{1+r 10^{p H-p K_{h 1}}}{1+10^{p H-p K_{h 1}}+10^{2 p H-p K_{h 1}-p K_{h 2}}}
$$

With this model, a satisfactory curve-fitting of the $\mathrm{A}(507 \mathrm{~nm})$ vs $\mathrm{pH}$ plot was obtained (Figure $\mathbf{5}, R=0.998$ ) with the following optimized parameters: $\mathrm{p} K_{h 1}=2.84( \pm 0.11), \mathrm{p} K_{h 2}=5.19( \pm 0.05)$, and $r=0.80( \pm 0.01)$. From the $r$ value, it can be noted that AHB is only $20 \%$ less colored than $(\mathrm{AH})_{2}$ despite the loss of one chromophore out of two. Thus, the hemiketal unit (B), although colorless, seems to participate in the residual color of AHB by imposing on the colored unit $(\mathrm{AH})$ a higher molar absorption coefficient than in $(\mathrm{AH})_{2}$. Hence, although the $\mathrm{p} K_{h l}$ value is close to the one of M3G $\left(\mathrm{p} K_{h}=2.83(24)\right.$ ), the discoloration at wine $\mathrm{pH}$ is much weaker owing to the fact that AHB retains $80 \%$ of the color potential of PA1 (fully expressed at $\mathrm{pH} 1$ ).

Semiempirical quantum mechanics calculations showed that water addition on the D-ring is more favorable than on the C-ring (Figure 4), the former hemiketal being more stable than the latter by ca. $2.2 \mathrm{kcal} \mathrm{mol}^{-1}$.

Regarding PA2, the $\mathrm{pH}$ dependence of the absorbance values at 505 and $590 \mathrm{~nm}$ is consistent with the existence of a simple acid-base equilibrium between the pyranoflavylium ion $(\mathrm{AH})$ and the quinonoid bases (A) without significant hydration (Figures 6 and 7). Consistently, Oliveira et al. (25) found that the simple pyranomalvidin 3-glucoside vitisin B does not undergo hydration in the $\mathrm{pH}$ range $0-8$ but only proton-transfer reactions. The $\mathrm{p} K_{\mathrm{a}}$ values of PA2 deduced from analysis at 505 and $590 \mathrm{~nm}$ (using a simplified version of eq 1 for a single $\mathrm{pH}$-dependent equilibrium) were coherent and, respectively, equal to $2.86( \pm 0.09)$ and $2.89( \pm 0.12)$. In addition, the following spectral parameter was obtained: $r_{\mathrm{A}}=\varepsilon_{\mathrm{A}} / \varepsilon_{\mathrm{AH}}=0.51( \pm 0.02)$ and $4.63( \pm 0.88)$ at 505 and $590 \mathrm{~nm}$, respectively. At $\mathrm{pH} \geq \mathrm{p} K_{a}$, the solution showed a remarkable increase in the values of the chroma (Figure 2), which is in agreement with the formation of the colored quinonoid bases.

Semiempirical quantum mechanics calculations show that the two tautomeric quinonoid bases A7 and A4' formed by respective deprotonation of $\mathrm{O} 7-\mathrm{H}$ and $\mathrm{O}^{\prime}-\mathrm{H}$ (Figure 7) have close energies (deprotonation of $\mathrm{O} 7-\mathrm{H}$ favored by only $0.6 \mathrm{kcal} \mathrm{mol}^{-1}$ ) so that the $K_{\mathrm{a}}$ value probably refers to an overall acidity constant for the formation of both bases. By contrast, the quinonoid base formed by deprotonation of the E-ring's phenolic $\mathrm{OH}$ is less stable than A7 by $3.6 \mathrm{kcal} \mathrm{mol}^{-1}$ and probably makes no significant contribution to $K_{\mathrm{a}}$. By comparison with M3G (24), it can be noted that PA2 is more acidic by ca. one $\mathrm{p} K_{\mathrm{a}}$ unit.

The presence of the additional methyl group in the PA2 structure exerts a decisive influence on the behavior of the pyranoanthocyanin in aqueous solution. On the one hand, it could destabilize noncovalent dimers by steric repulsion. On the other hand, it could prevent the binding of metal traces, which, in interaction with the catechol nucleus of PA1, could favor dimerization. However, with the visible spectrum of PA1 being essentially insensitive to the addition of excess of EDTA, we were unable to confirm this latter hypothesis.

Relative Stability in Aqueous Solution. Both pigments showed high stability. After 6 weeks, $63 \%$ of the initial PA 1 concentration remained in solution versus $72 \%$ for PA2. It should be noted that, in the same experimental conditions, only $40 \%$ of $\mathrm{M} 3 \mathrm{G}$ was recovered (32). After 6 weeks, no peaks with absorption in the visible region were detected in the chromatograms, and only small

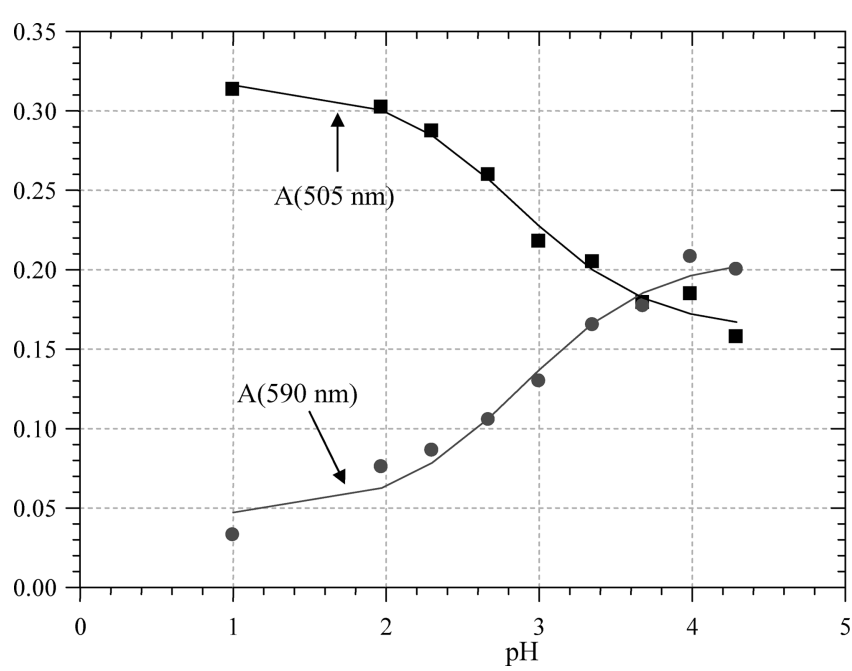

Figure 6. Changes in the absorbance at 505 and $590 \mathrm{~nm}$ of PA2 solutions at different $\mathrm{pH}$ values (concentration of pigments, $10^{-4} \mathrm{M}$ ).

additional peaks were detected at $280 \mathrm{~nm}$. It can be concluded that the conversion of anthocyanins into pyranoanthocyanins hinders not only the reversible formation of colorless forms but also the irreversible degradation of the pigments (see $\mathrm{pH}$ and relative stability assays).

Interaction with $(+)$-Catechin. The $\pi$-stacking binding between the pyranoanthocyanins and $(+)$-catechin, a common wine copigment, was investigated at $\mathrm{pH} 3.5$.

The addition of catechin to the PA1 solution induces a weak hyperchromic shift in the visible absorption band, which suggests a small displacement of the hydration equilibrium toward the pyranoflavylium ion due to its higher affinity for catechin (Figure 8). Only a weak bathochromic effect could be evidenced. At the $\mathrm{pH}$ considered, the second hydration equilibrium is negligible. The apparent copigmentation binding constant was evaluated with the assumption that catechin selectively binds the dimeric pyranoflavylium ion $(\mathrm{AH})_{2}$ (negligible binding to the heterodimer $\mathrm{AHB}$ ). From this simple model, the following equation can be readily derived $\left(A_{0}=\right.$ visible absorbance in the absence of copigment; $r_{\mathrm{CP}}=$ $\varepsilon_{\text {complex }} / \varepsilon_{\mathrm{AH} 2)}$ :

$$
A=A_{0} \frac{\left(1+r 10^{p H-p K_{h 1}}+r_{C P} K C P_{t}\right)\left(1+10^{p H-p K_{h 1}}\right)}{\left(1+10^{p H-p K_{h 1}}+K C P_{t}\right)\left(1+r 10^{p H-p K_{h 1}}\right)}
$$

Using the $r$ and $K_{h l}$ values previously determined and setting $r_{\mathrm{CP}}$ at a fixed value of 6.4 (see validation of this assumption in the analysis of the PA2-catechin copigmentation below), a curvefitting of $\mathrm{A}(507 \mathrm{~nm})$ versus total copigment concentration against eq 2 yielded a $K$ value of $17.1( \pm 0.7) \mathrm{M}^{-1}(R=0.99)$, which is weaker than that estimated for $\mathrm{M} 3 \mathrm{G}$ in the same conditions (89 $\mathrm{M}^{-1}(38)$ ). If we assume that pyranoanthocyanins should form more stable copigmentation complexes than the corresponding anthocyanins because of the wider planar polarizable chromophore of the former, the lower $K$ value actually obtained could be ascribed to the competition between copigmentation and noncovalent dimerization, which probably makes the pyranoflavylium ion of PA1 less available for catechin. Hence, through this crude model, we suggest that catechin binds to PA1 with concomitant dissociation of the dimer (in agreement with the low apparent binding constant) to form only very minor concentrations of the copigmentation complex. Our results are consistent with the previous observation that the 8,8-methylmethine catechin-M3G pigments, which also seem to have a strong propensity for noncovalent dimerization, only weakly bind chlorogenic acid, a potent anthocyanin copigment (24). 


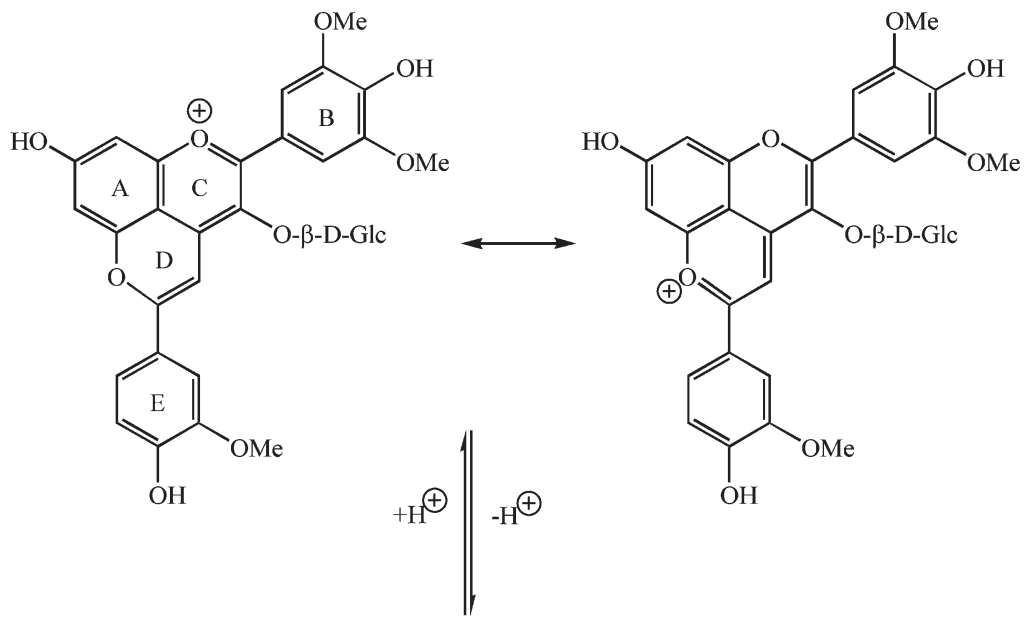

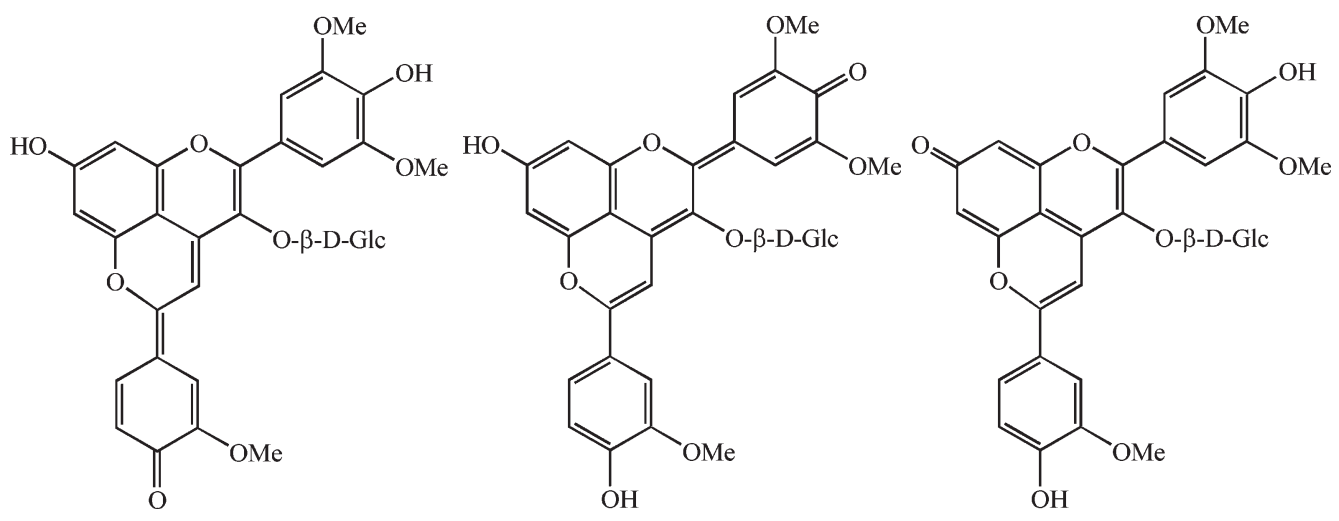

Figure 7. Proton transfer equilibria of PA2.

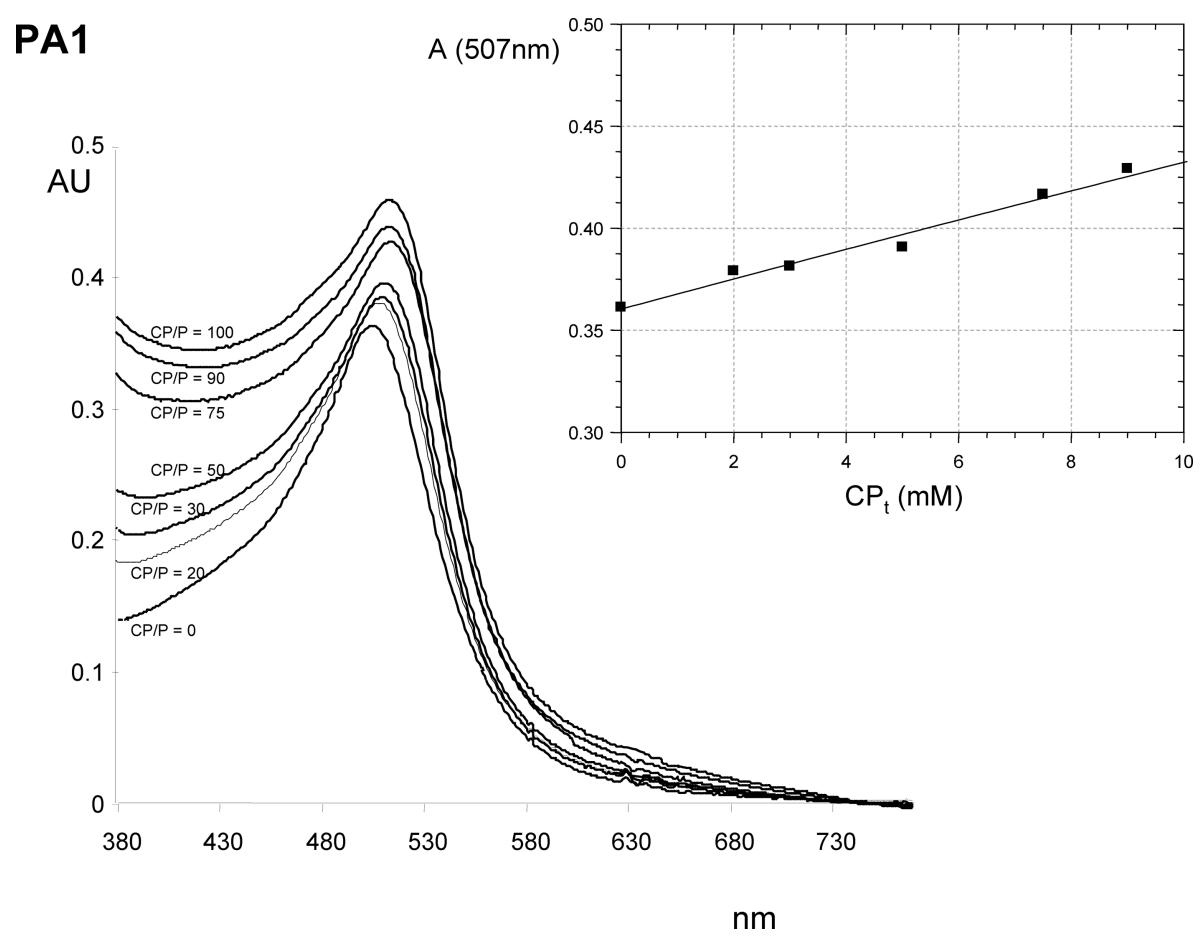

Figure 8. Visible spectra of PA1 solutions after the addition of $(+)$-catechin for different copigment/pigment molar ratio (CP/P) ranging from 0 to 100 . Inset: absorbance at $507 \mathrm{~nm}$ vs $(+)$-catechin concentration curve $\left(\mathrm{pH} 3.5,25^{\circ} \mathrm{C}\right)$.

PA2 binding to catechin promotes a spectacular increase in band I (with a concomitant slight decrease in band II) (Figure 9). This strong hyperchromic effect can be quantitatively interpreted by assuming a selective $\mathrm{AH}$-catechin binding (negligible binding between $\mathrm{A}$ and catechin) resulting in a copigmentation complex with a much higher molar absorption coefficient than free $\mathrm{AH}$. For estimating the binding constant, eq 2 was used, with $r$ being replaced by $r_{\mathrm{A}}$ and $K_{h l}$ by $K_{a}$. A curve-fitting of the $\mathrm{A}(505 \mathrm{~nm})$ 


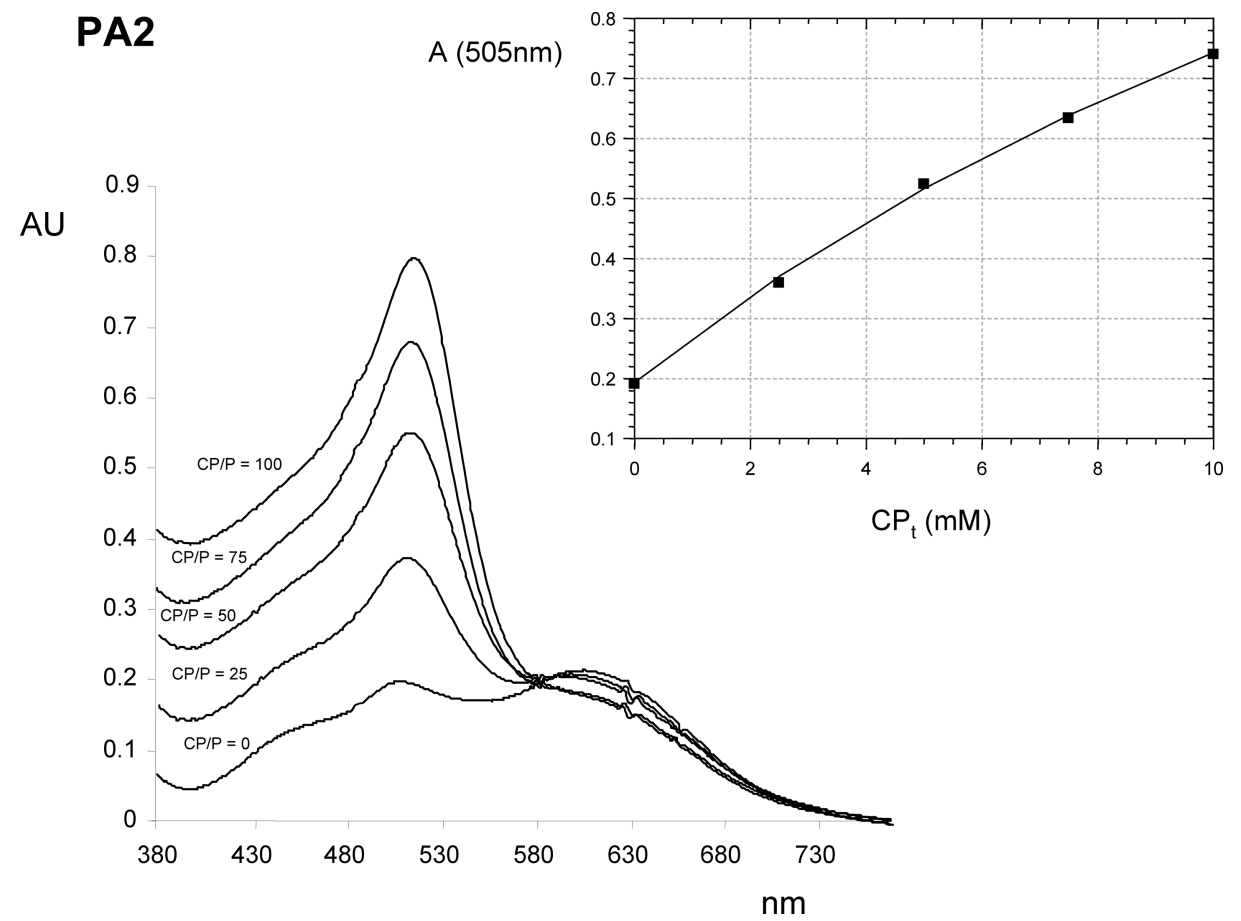

Figure 9. Visible spectra PA2 solutions after the addition of $(+)$-catechin for different copigment/pigment molar ratios $(\mathrm{CP} / \mathrm{P})$ ranging from 0 to 100 . Inset: absorbance at $505 \mathrm{~nm}$ vs $(+)$-catechin concentration curve $\left(\mathrm{pH} 3.5,25^{\circ} \mathrm{C}\right)$.

versus catechin concentration curve using the $K_{\mathrm{a}}$ and $r_{\mathrm{A}}$ parameters determined previously gave the following optimized parameters: $K=228( \pm 40)$ and $r_{\mathrm{CP}}=\varepsilon_{\mathrm{AHCP}} / \varepsilon_{\mathrm{AH}}=6.4( \pm 0.8)(R=$ $0.9995)$. The $K$ value obtained is consistent with our interpretation that copigmentation may well be stronger with pyranoanthocyanins than with anthocyanins, provided that the latter are not strongly self-associated as is the case for PA1. Moreover, the huge increase in the absorption of the pyranoflavylium ion of PA2 when it binds catechin has no equivalent for anthocyanins and nicely outlines the potential of pyranoanthocyanins in the expression of natural colors.

Colorimetric Effects of Copigmentation. Tristimulus colorimetry enabled us to establish the variation in the chromatic characteristics of the copigmented solutions of PA1 and PA2. As previously observed in copigmentation essays carried out with $\mathrm{M} 3 \mathrm{G}$ and different flavonoids (39), the addition of the copigment induced a decrease in lightness $L^{*}$ and an increase in hue $h_{\mathrm{ab}}$ and chroma $C_{\text {ab }}^{*}$ (Figure 10). Thus, the hyperchromic effect produced by copigmentation gives rise to a darkening and a more intense color of the solutions.

The intensity of the changes observed in chroma and hue angle were quite different. The modifications in $h_{\mathrm{ab}}$ were much higher in PA2 than in PA1 (99 and 11 CIELAB units, respectively). As the concentration of $(+)$-catechin increases, the solutions of PA2 shift from purple toward red and from red toward reddish in those of PA1. Also, the modifications in chroma, the quantitative attribute of colorfulness, were higher in the solutions with PA2.

Color differences, $\Delta E^{*}{ }_{\mathrm{ab}}=\left[\left(\Delta L^{*}\right)^{2}+\left(\Delta a^{*}\right)^{2}+\left(\Delta b^{*}\right)^{2}\right]^{1 / 2}$, between the copigmented solutions and the noncopigmented ones were also calculated (Table 2). In general, the eye is able to discriminate two colors when $\Delta E^{*}{ }_{\text {ab }} \geq 1(40,41)$, but when the wine is observed through standardized winetasting glasses, the color discrimination is weaker, and the acceptable tolerance for the human eye in distinguishing the color of wines is 3.0 CIELAB units (42). Color differences between PA2 solutions copigmented with $(+)$-catechin and the PA2 control solution were always easily detectable by the human eye since $\Delta E^{*}$ ab values were always

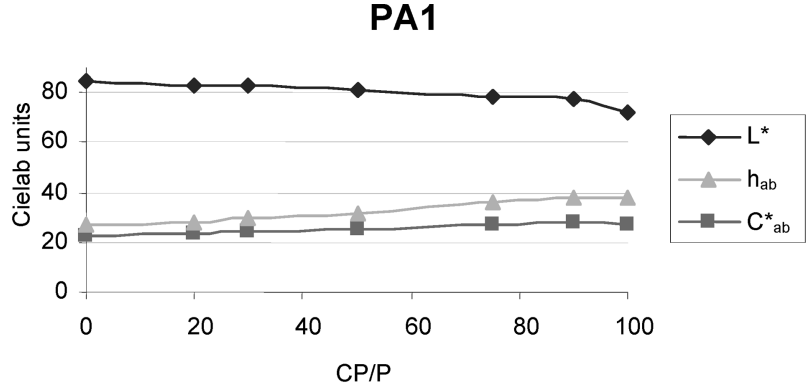

PA2

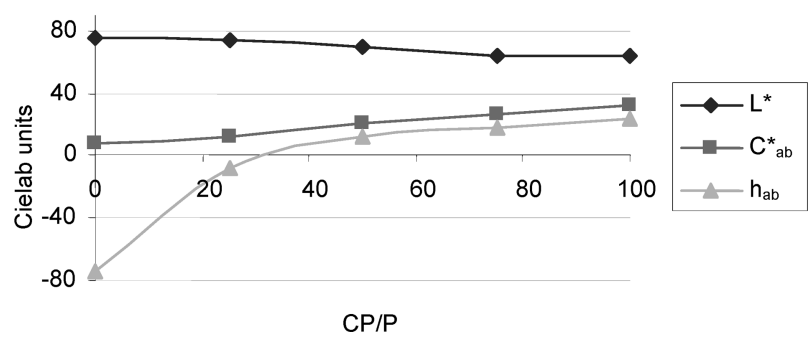

Figure 10. Changes in the lightness $\left(L^{*}\right)$, chroma $\left(C^{*}\right.$ ab $)$, and hue angle $\left(h_{\mathrm{ab}}\right)$ of solutions of PA1 and PA2 after the addition of increasing concentrations of $(+)$-catechin (copigment/pigment molar ratio ranging from 0 to 100).

much higher than 3 units. In contrast, a (+)-catechin/PA1 molar ratio higher than 50 was needed to obtain noticeable color changes in the solution. Therefore, copigmentation caused more intense chromatic changes in PA2 solutions than in PA1.

In conclusion, the pyranoanthocyanins formed by the reaction of the main wine anthocyanin malvidin 3-glucoside with vinylcatechol (PA1) or vinylguaiacol (PA2) display unexpectedly discriminated color properties. The additional methyl group of PA2 cancels the noncovalent dimerization of the pyranoflavylium ions and favors proton transfer over hydration. Thereby, it enhances the propensity of the pyranoflavylium ion to bind the common 
Table 2. Color Differences $\left(\Delta E^{*}{ }_{a b}\right)$ between the Reference Solutions of PA1 and PA2 (Noncopigmented) and the Solutions Added with Increasing Concentrations of ( + )-Catechin (Copigment/Pigment Molar Ratio Ranging from 25 to 100)

\begin{tabular}{lcc}
\hline $\mathrm{CP} / \mathrm{P}$ & $\frac{\mathrm{PA} 1}{\Delta E_{\mathrm{ab}}^{*}}$ & $\frac{\mathrm{PA} 2}{\Delta E^{*}}$ \\
\hline 25 & 2.0 & 11.3 \\
50 & 2.8 & 22.3 \\
75 & 6.7 & 29.8 \\
100 & 9.7 & 35.7 \\
\hline
\end{tabular}

wine copigment catechin with a simultaneous huge increase in the molar absorption coefficient at the wavelength of maximal visible absorption. These results suggest that pyranoanthocyanins may contribute to the color of aged wines in a very discriminated way, depending on the substituents of their additional aromatic rings.

\section{LITERATURE CITED}

(1) Mateus, N.; de Freitas, V. Evolution and stability of anthocyaninderived pigments during Port wine aging. J. Agric. Food Chem. 2001, 49, 5217-5222.

(2) Hayasaka, Y.; Asenstorfer, R. E. Screening for potential pigments derived from anthocyanins in red wine using nanoelectrospray tandem mass spectrometry. J. Agric. Food Chem. 2002, 50, 756-761.

(3) Fulcrand, H.; Dueñas, M.; Salas, E.; Cheynier, V. Phenolic reactions during winemaking and aging. Am. J. Enol. Vitic. 2006, 57, 289-297.

(4) Monagas, M.; Martin-Alvarez, P. J.; Bartolome, B.; Gomez-Cordoves, C. Statistical interpretation of the color parameters of red wines in function of their phenolic composition during aging in bottle. Eur. Food Res. Technol. 2006, 222, 702-709.

(5) Boido, E.; Alcalde-Eon, C.; Carrau, F.; Dellacassa, E.; Rivas-Gonzalo, J. C. Aging effect on the pigment composition and color of Vitis vinifera L. Cv. Tannat wines. Contribution of the main pigment families to wine color. J. Agric. Food Chem. 2006, 54, 6692-6704.

(6) Fulcrand, H.; Benabdeljalil, C.; Rigaud, J.; Cheynier, V.; Moutounet, $\mathrm{M}$. A new class of wine pigments generated by reaction between pyruvic acid and grape anthocyanins. Phytochemistry 1998, 47, 1401-1407.

(7) Romero, C.; Bakker, J. Interactions between grape anthocyanins and pyruvic acid, with effect of $\mathrm{pH}$ and acid concentration on Anthocyanin composition and color in model solutions. J. Agric. Food Chem. 1999, 47, 3130-3139.

(8) Bakker, J.; Timberlake, C. F. Isolation, identification, and characterization of new color-stable anthocyanins occurring in some red wines. J. Agric. Food Chem. 1997, 45, 35-43.

(9) Benabdeljalil, C.; Cheynier, V.; Fulcrand, H.; Hakiki, A.; Mosaddak, M.; Moutounet, M. Mise en évidence de nouveaux pigments formés par réaction des anthocyanes avec des métabolites de levures. Sci. Aliments 2000, 20, 203-220.

(10) Fulcrand, H.; Cameira-Dos-Santos, P. J.; Sarni-Manchado, P.; Cheynier, V.; Favre Bonvin, J. Structure of new anthocyanin-derived wine pigments. J. Chem. Soc. Perkin Trans. 1 1996, 735-739.

(11) Hakansson, A. E.; Pardon, K.; Hayasaka, Y.; De Sa, M.; Herderich, M. Structures and colour properties of new red wine pigments. Tetrahedron Lett. 2003, 44, 4887-4891.

(12) Francia-Aricha, E. M.; Guerra, M. T.; Rivas-Gonzalo, J. C.; SantosBuelga, C. New anthocyanin pigments formed after condensation with flavanols. J. Agric. Food Chem. 1997, 45, 2262-2266.

(13) Mateus, N.; Carvalho, E.; Carvalho, A. R.; Melo, A.; GonzálezParamás, A. M.; Santos-Buelga, C.; Silva, A.; Freitas, V. Isolation and structural characterization of new acylated anthocyanin-vinylflavanol pigments occurring in aging red wines. J. Agric. Food Chem. 2003, 51, 277-282.

(14) Lu, Y.; Foo, L. Y. Unusual anthocyanin reaction with acetone leading to pyranoanthocyanin formation. Tetrahedron Lett. 2001, 42, 1371-1373.

(15) He, J.; Santos-Buelga, C.; Silva, A. M. S.; Mateus, N.; De Freitas, V. Isolation and structural characterization of new anthocyanin-derived yellow pigments in aged red wines. J. Agric. Food Chem. 2006, 54, 9598-9603.

(16) Schwarz, M.; Wabnitz, T. C.; Winterhalter, P. Pathway leading to the formation of anthocyanin-vinylphenol adducts and related pigments in red wines. J. Agric. Food Chem. 2003, 51, 36823687.

(17) Alcalde-Eon, C.; Escribano-Bailon, M. T.; Santos-Buelga, C.; RivasGonzalo, J. C. Changes in the detailed pigment composition of red wine during maturity and ageing. A comprehensive study. Anal. Chim. Acta 2006, 563, 238-254.

(18) Brouillard, R.; Dubois, J. E. Mechanism of the structural transformations of anthocyanins in acidic media. J. Am. Chem. Soc. 1977, 99, $1359-1364$.

(19) Brouillard, R.; Delaporte, B. Chemistry of anthocyanin pigments. 2. Kinetic and thermodynamic study of proton transfer, hydration, and tautomeric reactions of malvidin 3-glucoside. J. Am. Chem. Soc. 1977, 99, 8461-8468.

(20) Brouillard, R.; Lang, J. The hemiacetal-cis-chalcone equilibrium of malvin, a natural anthocyanin. Can. J. Chem. 1990, 68, 755-61.

(21) Brouillard, R.; Dangles, O. Anthocyanin molecular-interactions the first step in the formation of new pigments during wine aging. Food Chem. 1994, 51, 365-371.

(22) Goto, T.; Kondo, T. Structure and Molecular Stacking of Anthocyanins. Flower Color Variation. Angew. Chem., Int. Ed. 1991, 30, 17-33.

(23) Escribano-Bailón, T.; Dangles, O; Brouillard, R. Coupling reactions between flavylium ions and catechin. Phytochemistry 1996, 41, 1583-1592.

(24) Duenas, M.; Salas, E.; Cheynier, V.; Dangles, O.; Fulcrand, H. UVvisible spectroscopic investigation of the 8,8-methylmethine catechin-malvidin 3-glucoside pigments in aqueous solution: Structural transformations and molecular complexation with chlorogenic acid. J. Agric. Food Chem. 2006, 54, 189-196.

(25) Oliveira, J.; Mateus, N.; Silva, A. M. S.; Freitas, V. Equilibrium forms of vitisin B pigments in an aqueous system studied by NMR and visible spectroscopy. J. Phys. Chem. B 2009, 113, 11352-11358.

(26) Heredia, F. J.; Francia Aricha, E. M.; Rivas Gonzalo, J. C.; Vicario, I. M.; Santos Buelga, C. Chromatic characterization of anthocyanins from red grapes I. pH effect. Food Chem. 1998, 63, 491-498.

(27) Alcalde-Eon, C.; Escribano-Bailon, M. T.; Santos-Buelga, C.; RivasGonzalo, J. C. Separation of pyranoanthocyanins from red wine by column chromatography. Anal. Chim. Acta 2004, 513, 305-318.

(28) Wang, H.; Race, E. J.; Shrikhande, A. J. Anthocyanin transformation in Cabernet Sauvignon wine during aging. J. Agric. Food Chem. 2003, 51, 7989-7994.

(29) Boido, E.; Alcalde-Eon, C.; Carrau, F.; Dellacassa, E.; Rivas-Gonzalo, J. C. Aging effect on the pigment composition and color of Vitis vinifera L. Cv. Tannat wines. Contribution of the main pigment families to wine color. J. Agric. Food Chem. 2006, 54, 6692-6704.

(30) Heredia, F. J.; Álvarez, C.; González-Miret, M. L.; Ramírez, A. CromaLab, análisis de color; Registro General de la Propiedad Intelectual SE-1052-04: Sevilla, Spain, 2004.

(31) CIE. Colorimetry, 2nd ed.; Publication 15.2; CIE: Vienna, Austria, 1986.

(32) Escribano-Bailon, T.; Alvarez-Garcia, M.; Rivas-Gonzalo, J. C.; Heredia, F. J.; Santos-Buelga, C. Color and stability of pigments derived from the acetaldehyde-mediated condensation between malvidin 3-O-glucoside and (+)-catechin. J. Agric. Food Chem. 2001, 49, 1213-1217.

(33) Brouillard, R.; Delaporte, B. Chemistry of anthocyanin pigments. 2. Kinetic and thermodynamic study of proton transfer, hydration, and tautomeric reactions of malvidin 3-glucoside. J. Am. Chem. Soc. 1977, 99, 8461-8468.

(34) Bakker, J.; Timberlake, C. F. Isolation, identification, and characterization of new color-stable anthocyanins occurring in some red wines. J. Agric. Food Chem. 1997, 45, 35-43.

(35) Oliveira, J.; Fernandes, V.; Miranda, C.; Santos-Buelga, C.; Silva, A.; Freitas, V.; Mateus, N. Color properties of four cyanidin-pyruvic acid adducts. J. Agric. Food Chem. 2006, 54, 6894-6903.

(36) Brouillard, R.; Dubois, J. E. Mechanism of the structural transformations of anthocyanins in acidic media. J. Am. Chem. Soc. 1977, 99 , 1359-1364.

(37) Berké, B.; Chèze, C.; Vercauteren, J.; Deffieux, G. Bisulfite addition to anthocyanins: revisited structures of colourless adducts. Tetrahedron Lett. 1998, 39, 5771-5774.

(38) Malien-Aubert, C.; Dangles, O; Amiot, M. J. Influence of procyanidins on the color stability of oenin solutions. J. Agric. Food Chem. 2002, 50, 3299-3305. 
(39) Gómez-Miguez, M.; González-Manzano, S.; Escribano-Bailón, M. T.; Heredia, F. J.; Santos-Buelga, C. Influence of different phenolic copigments on the color of malvidin 3-glucoside. J. Agric. Food Chem. 2006, $54,5422-5429$.

(40) Gonnet, J. F. Colour effects of copigmentation of anthocyanins revisiteds1. A colorimetric definition using CIELAB scale. Food Chem. 1998, 63, 409-415.

(41) Negueruela, A. I.; Echávarri, J. F.; Ayala, F.; Lomas, A. M. Colorimetría en vinos. Zubía monográfico 1995, 7, 151-166.
(42) Martínez, J. A.; Melgosa, M.; Pérez, M. M.; Hita, E.; Negueruela, A. I. Note: Visual and instrumental color evaluation in red wines. Food Sci. Technol. Int. 2001, 7, 439-444.

Received for review March 26, 2010. Revised manuscript received July 21, 2010. Accepted July 22, 2010. Thanks are due to the Spanish MICINN (Project ref AGL2008-05569-C02-01) and to Junta de Castilla y León (group GR133) for financial support. N.Q.-M. thanks the Spanish MICINN for the FPI predoctoral scholarship. 